\title{
Improving functional and environmental performance of Portland cement-based materials by graphene nanostructures
}

\author{
Vladimir Pershin ${ }^{1 *}$, Ali Mashhadani ${ }^{2}$, Denis Melekhin ${ }^{3}$, and Aleksey Osipov ${ }^{1}$ \\ ${ }^{1}$ Department of Technology and Methods of Nanoproducts Manufacturing, Tambov State Technical \\ University, 106 Sovetskaya Street, 392000, Tambov, Russian Federation \\ ${ }^{2}$ Post-graduate student from the Republic of Iraq, Tambov State Technical University, 106 \\ Sovetskaya Street, 392000, Tambov, Russian Federation \\ ${ }^{3}$ Architecture Faculty, Czech Technical University in Prague, 1903/4 Zikova, 16636 Prague 6 Czech \\ Republic
}

\begin{abstract}
Nanoparticles can accelerate cement hydration due to their high activity compacting microstructure, and consequently increase the strength. For the industrial production of concrete modified with nanoscale particles, it is necessary to develop an environmentally friendly and cheap technology for the industrial production of nanoparticles. The article presents the results of increasing the operational characteristics of concrete by adding graphene nanostructures obtained by liquid-phase shear exfoliation of graphite. This environmentally friendly technology requires energy costs, at least ten times less than technologies that use ultrasonic treatment of the suspension. Our industrial partner set a specific task: to find the minimum concentration of graphene structures in relation to cement, at which the compressive strength of cement-sand mix increases by $30 \%$. It was experimentally established that at concentrations of graphene nanostructures with respect to cement not more than $0.02 \%$, the compressive strength increases by at least $30 \%$.
\end{abstract}

\section{Introduction}

Concrete is the most used material in the global construction industry, but so far there are a number of problems: insufficient strength, especially when bending; high absorption of moisture and therefore not sufficient durability; high carbon dioxide emissions from cement production. Of course, there are other disadvantages of concrete, but in our opinion these are the three main disadvantages that need to be urgently addressed. There is a wide consensus in the research community that concrete should be developed on a nanoscale, where its chemical and physiomechanical properties can really be improved [1]. Nanoparticles can accelerate cement hydration due to their high activity, densifying the microstructure and, therefore, increasing strength and durability [2]. By introducing

*Correspondingauthor:pershin.home@mail.ru 
engineering nanomaterials, such as nano- $\mathrm{SiO}_{2}$, nano- $\mathrm{Fe}_{2} \mathrm{O}_{3}$, nano- $\mathrm{Al}_{2} \mathrm{O}_{3}$, nano- $\mathrm{CuO}$, nano$\mathrm{TiO}_{2}$, it is possible to increase the mechanical properties and durability of concrete [2].

The effect of nanometakaolin (NMK) on the mechanical properties and microstructure of Portland cement mortar was investigated [3]. The NMK was prepared by thermal activation of kaolin clay for 2 hours at $750^{\circ} \mathrm{C}$. Based on experimental studies, the authors made the following conclusions:

- Compressive and tensile strength of the cement mortars with NMK is higher than that of the plain cement mortar with the same w/b ratio.

- The enhancement of tensile strength was $49 \%$ above control mortar; whereas the enhancement of compressive strength was $7 \%$ at $8 \%$ NMK replacement.

- The NMK in cement mortar acts as a nano-fiber due to its morphology.

- The SEM observations confirmed that the NMK was not only acting as filler, but also as an activator to promote hydration process.

Many articles are devoted to studies of the effect of graphene oxide and graphene on concrete performance [4-10]. Graphene oxide (GO), which has been successfully used in cement composites as an admixture, can improve their mechanical and durability properties [4]. This study examined and elucidated the effect of GO on the hydration products of a cement system in detail. The hydration precursors, morphology, composition, and chemical bonds of cement pastes were studied by SEM/BSE, XRD, LA-ICP-MS, and 29Si/27Al MAS-NMR, respectively. The experimental results indicated that the 28-day compressive strength of cement pastes (w/c 1/4 0.35) was increased by $29 \%$ when admixing $0.02 \mathrm{wt} \%$ GO. There existed chemical reactions between the admixed GO and the cement hydration products. The improved strength actually was not only attributed to the improved hydration degree of cement, but also the newly found tobermorite-like hydrates and jennite-like hydrates due to the $\mathrm{Ca}$ ions consumption by the negatively charged GO. The polymerization of hydration products was also improved due to the addition of GO. The incorporation of $0.16 \mathrm{wt} \% \mathrm{GO}$ into the cement matrix can enhance the flexural strength of the material by $11.62 \%$ due to the higher hydration degree, nano-filler effect and crackingbridging effect [5].

The rate of hydration accelerated and the cumulative hydration heat increased with the increasing proportions of GO in the composite [6]. GO dosages about 0.02 and $0.04 \mathrm{wt} \%$ of cement in the composites resulted the maximum enhancement of compressive and flexural strength by 83 and $26 \%$, respectively, compared to the control mix ( 0 wt \% GO). The microstructural investigation shows that GO enhanced the hydration of calcium hydroxide $(\mathrm{CH})$ and calcium silicate hydrate $(\mathrm{C}-\mathrm{S}-\mathrm{H})$ during the nucleation and growth stages, filled pores, bridged micro-cracks and created interlocking between the cement hydration products. Collectively, these effects ultimately improved the mechanical properties of the composites. Also, in this process, the 0.02 and $0.04 \mathrm{wt} \%$ GO cement composite increased the electrical resistivity by $11.5 \%$, and decreased the absorptivity by $29 \%$, respectively, both of which improved the overall performance of the composite.

The study [7] examines and compares the performance of two specific forms of graphene nanomaterials in the cement based composite, namely graphene oxide (GO) and reduced graphene oxide (rGO). The effect of GO and rGO on workability, early age hydration, microstructure, mechanical and transport properties was determined. The XRD and TGA quantification of the hydration products shows that GO composites have a greater content of $\mathrm{Ca}(\mathrm{OH})_{2}$ and $\mathrm{C}-\mathrm{S}-\mathrm{H}$ compared to $\mathrm{rGO}$ composites measured at 1,7 and 28 days. Meso and gel pores $(<2 \mathrm{~nm})$ related to $\mathrm{C}-\mathrm{SH}$ show increasing trend in the GO and rGO composites compared to the control mix in dynamic vapour sorption (DVS) analysis. Compared to the control mix, the 28 -day compressive strength increased by $10 \%$ and $15 \%$ in $0.06 \mathrm{wt} \%$ of GO and $\mathrm{rGO}$ in the composites. However, the highest flexural strength was 
increased in the $0.04 \mathrm{wt} \%$ of $\mathrm{GO}$ and $0.06 \mathrm{wt} \% \mathrm{rGO}$ composite, and the enhancement was $75.7 \%$ and $33.7 \%$, respectively, compared to the control mix.

The effects of graphene oxide (GO) on the hydration kinetics, pore structure, mechanical properties, and the structure of calcium silicate hydrate $(\mathrm{C}-\mathrm{S}-\mathrm{H})$ gels were systematically investigated by combinatorial techniques [8]. GO can accelerate the cement hydration, refine the pore structure and increase the polymerization degree of C-S-H gels due to the nucleation effects of GO. The specific surface area of cement paste and the number of gel pores were reduced with the addition of GO. A 3D network structure of GO modified C-S-H gels was originally proposed based on the obtained results that GO could intercalate into the interlayer space of C-S-H gels through ionic bonding with $\mathrm{Ca}^{2+}$ and fill in the gel pores. A small fraction of GO $(0.022 \mathrm{wt} \%)$ increased the $28 \mathrm{~d}$ compressive strength by $16.31 \%-25.60 \%$ at a various water to cement ratios, indicating that GO is a potential nano-reinforcing material for cement composites.

Acid attack on cement concrete results in the development of a degraded layer surrounding the unaffected material, which causes a deterioration of mechanical properties. The effect of reduced graphene oxide, alumina and silica nanoparticles on the deterioration characteristics of 28-day cured cementitious pastes after storage in $0.5 \mathrm{moL} / \mathrm{L} \mathrm{HNO} 3$ solution for a period of 56 days is reported [9]. The porosimetry results showed that the presence of reduced graphene oxide and nano alumina decreased the amount of capillary pores $(10 \mathrm{~nm}-10 \mu \mathrm{m})$ by up to $46 \%$ and $51 \%$ than the control paste. Overall, the results suggest that the presence of these nanomaterials refined the pore structure of the cementitious matrix and thereby increased the resistance to leaching of calcium ions from the binder phases exposed to aggressive aqueous media.

To further delineate the collective effects of GNPs and GONPs, particularly their contents, on the performance of concrete, a comprehensive investigation is carried out in the study [10]. Mortar samples, characterized by different water-cement ratios and containing different amounts of GNPs and GONPs, are experimentally tested to systematically probe their strength, electrical resistance and piezoresistive reactions. With a proper water-cement ratio, a small percentage of GNPs and GONPs can significantly improve the concrete strength, while the content of GNPs needs to be substantially increased to achieve low electrical resistance and accurate piezoresistive reaction.

This article presents the results of the influence of GNP on the strength of a cementsand mixture, since the strength of concrete mainly depends on the strength of this mixture. In our opinion, the use of GNP for concrete modification on an industrial scale has better prospects than civil defense, for the following reasons: GNP production technology is environmentally friendly; no industrial waste; less energy consumption than in the production of GO. Moreover, our industrial partner set a specific task: to find the minimum concentration of graphene structures in relation to cement, at which the strength of cementsand mix increases by $30 \%$.

\section{Materials and experimental procedure}

\subsection{Materials}

To obtain few- layer graphene in this work, we used graphite grade GSM-2 according to GOST 18191-78 (Russian Standard) "Special low-ash graphite. Technical conditions" According to physic-chemical indicators, it meets the requirements indicated in the table. The choice of material is justified by its use at NanoTechCenter LLC (Tambov, Russia) for producing few-layer graphene by liquid-phase exfoliation by ultrasonic treatment of graphite oxide according to the Hamers-Ofemann method [5]. 
When preparing the suspension, water was used in accordance with GOST 23732-2011 (Russian standard) "Water for concrete and mortar. Technical conditions. " To prevent agglomeration of graphene nanostructures, the surfactant OP-7 was used according to GOST 8433-81 "Substances auxiliary OP-7 and OP-10. Technical conditions. " The concentration of OP-7 was $0.3 \%$, by weight of water. In addition, concrete modifiers were used as surfactants according to GOST R 56178-2014 "Organic-mineral modifiers MB type for concrete, mortar and dry mixes. Technical conditions".

\subsection{Experimental setup}

Many methods for producing graphene flakes are known [11], but we used the method of liquid-phase exfoliation of graphite $[1,12]$.

The apparatus consists of a cylindrical stator, a rotor and a rotor rotation drive. In contrast to the apparatus used in $[1,12]$, radial grooves in which movable blades were mounted were cut in the rotor. In addition, a cover was installed on the stator; holes were placed only in the upper part of the cylindrical shell. The blades were installed in such a way that under the action of centrifugal forces that arose during the rotation of the rotor, the blades moved in the radial direction and glided along the inner surface of the stator with almost no gap. The inner diameter of the stator is $40 \mathrm{~mm}$, and the height is $50 \mathrm{~mm}$. The rotor speed varied from 5,000 to 20,000 rpm.

Changes were made to our design for the following reasons. The authors of [1] believe that exfoliation occurs mainly when particles passing through holes in the stator are exposed to blades. The necessary conditions for the exfoliation of graphite particles are: the gap between the stator and the rotor blades should be no more than $0.1 \mathrm{~mm}$; the local shear rate in the zone of contact of the blades with the stator should not be less than $10 \mathrm{~s}$. In our design, exfoliation occurs in the gap between the blade and the inner surface of the stator. The cover provides a stable circulation loop for the movement of the suspension, which enters the processing zone only through the lower end of the stator, and exits through the holes. The location of the holes in the upper part of the stator provides the maximum residence time of particles in the contact zone of the blades with the inner surface of the stator.

Centrifugal forces act on the fluid and particles located in the area between the stator, the rotating rotor and adjacent blades. Under the action of these forces, the particles are pressed against the inner surface of the stator and the peripheral speed of their motion decreases. When a particle enters the zone between the stator and the moving blade, shear forces begin to act, as a result of which inside the particle, in addition to normal stresses, tangential stresses arise, which leads to a shift of the graphene layers relative to each other. Suppose that a particle consists of five graphene layers. As a result of a shift, two are formed from one particle. In this case, the following options are possible: 1 layer +4 layers; 2 layers +3 layers. Apparently, the probabilities of each of these options are the same.

\subsection{Methodology of the experiments}

Investigations of the exfoliation process were carried out as follows. Prepared 5 litters of an aqueous suspension of crystalline graphite GSM-2 with a concentration of 5 to $20 \%$, poured it into a cylindrical container, installed the apparatus and began processing the suspension. Every 10 minutes, the process was stopped and $100 \mathrm{ml}$ of suspension was taken. The suspension was centrifuged, the precipitate was removed, and the concentration of graphene nanostructures in the remaining suspension was determined. After analysis, the sample was poured into a container and the exfoliation process was continued. The process was continued until the increase in the concentration of graphene structures ceased. During the 
experiments, the rotor speed was changed: $5000 ; 10000 ; 12000 ; 15,000 ; 20,000 \mathrm{rpm}$ At the end of the exfoliation process, the suspension was centrifuged and the precipitate was removed. The purified suspension was used as mixing water in the preparation of concrete samples for compression and bending tests. These tests were carried out in accordance with GOST 310.4-81 "Cements. Methods of bending and compression strength determination". For testing, $40 \times 40 \times 160 \mathrm{~mm}$ beams were prepared. Three-point bending tests were carried out on the machine, and compression tests on the press.

The concentration of graphene structures ranged from 0 to $0.05 \%$ after $0.01 \%$ (relative to cement). Thus, we included few- layer graphene in a mixture of cement and sand, mixing water-based graphene dispersions with ordinary Portland cement (OPC) and fine dry sand. The cement / sand / water ratio was $1 / 2.7 / 0.53$. The fresh mixture was poured into standard steel molds measuring $40 \times 40 \times 160 \mathrm{~mm}$, subjected to vibration to remove air, removed from the steel molds after 24 hours and stored in a water tank for curing. Beams (Fig. 1) were tested for three-point bending (Fig. 2) after 7, 14 and 28 days of curing.

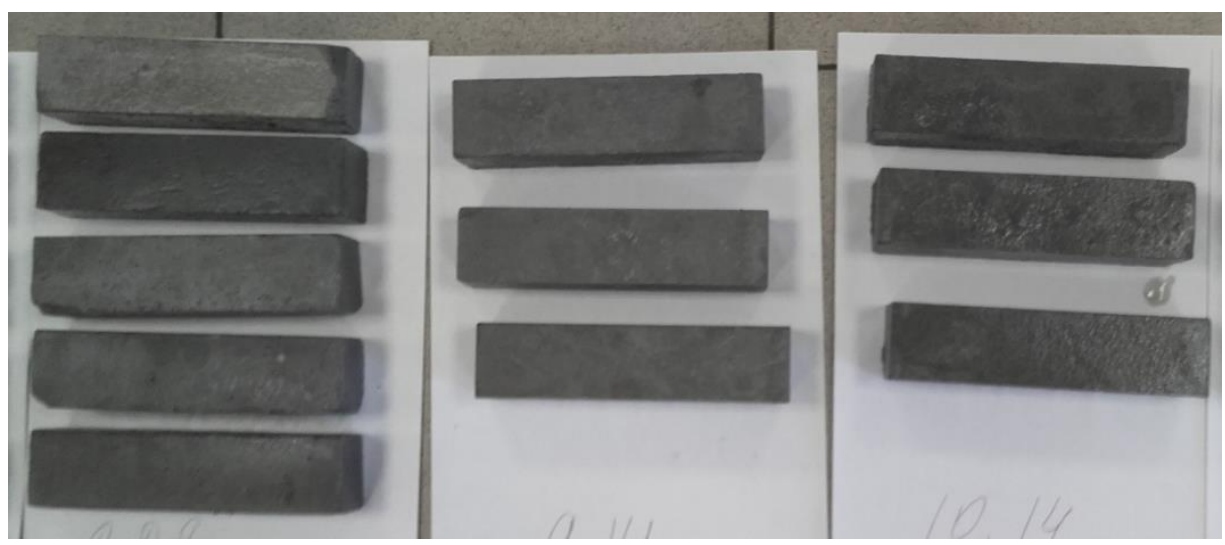

Fig. 1. Beams after curing.

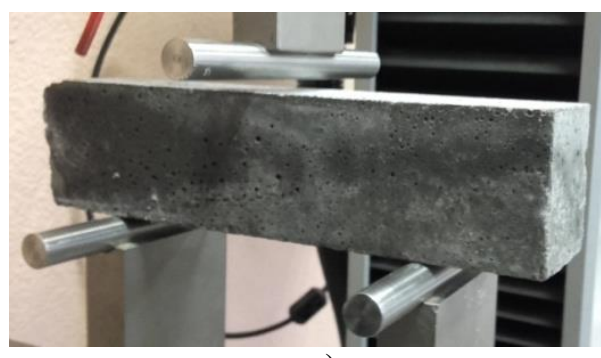

a)

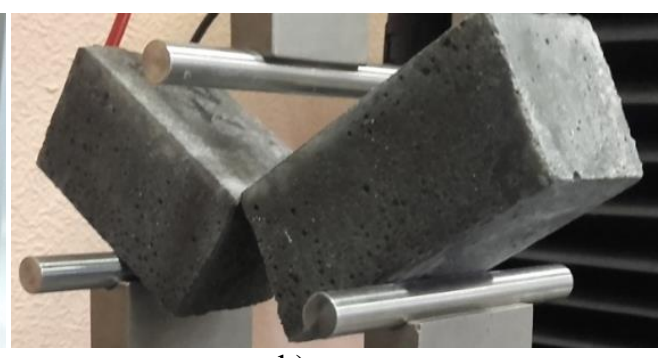

b)

Fig. 2. Tests of beams for three-point bending: $a$ - before testing; $b$ - after testing.

In addition, cubes were made from the halves of the beams after bending tests and tested for compression.

\section{Results and discussion}

Figure 3 shows the characteristic changes in bending strength for control beams and beams made of cement-sand mixture, modified with few-layer graphene. In this case, the concentration of few-layer graphene in water ranged from 0.25 to $0.3 \mathrm{mg} / \mathrm{ml}$. Considering that the water-cement ratio was 0.53 , the concentration of few-layer graphene in the cement-sand mixture ranged from 0.013 to $0.016 \%$. As you can see, the increase in bending 
strength during curing ( 7,14 and 21 days) is almost linear. Ultimately (after 28 days), the strength of the beams made of cement-sand mixture modified with few-layer graphene increased by $14 \%$, compared to control samples. This result agrees well with the data presented in the papers. As you can see, the increase in bending strength during the curing process (7, 14 and 28 days) is almost linear. Ultimately (after 28 days), the strength of the beams made of cement-sand mixture modified with few-layer graphene increased by $14 \%$, compared to control samples. This result agrees well with the data presented in $[1,5]$.

Figure 4 shows the change in compressive strength of control samples modified with graphene. You can see that the strength increases linearly, and ultimately for modified samples, the compressive strength is $33 \%$ higher than the strength of the control samples. Earlier [13], we experimentally found that when the concentration of few-layer graphene with respect to cement was $0.05 \%$, the compressive strength increased by about $60 \%$.

Thus, with an increase in the concentration of few-layer graphene by 3.5 times (from 0.014 to $0.05 \%$ ), the strength increases only 2 times.

It should be noted that in a number of experiments using a suspension with the same concentration of few-layer graphene we obtained an increase in strength by $50 \%$. Such a large discrepancy in the results ( 33 and $50 \%$ ) can probably be explained by the different quality of few-layer graphene, more precisely the average number of graphene layers in the particles that were in suspension after centrifugation. Indeed, to obtain better results, we processed the suspension in a rotary apparatus for a larger number of cycles and centrifuged for a longer time.

For industrial use of concrete modified with few-layer graphene, it is necessary to solve the problem of accurately determining the concentration of this graphene in suspension and the average number of layers. In our opinion, the main importance is not the concentration of few-graphene, but the number of particles per unit volume, since it is the particles that are the centers of formation of complexes on which the strength of concrete depends. In one liter of suspension, which contains particles with two graphene layers, there will be twice as many of these particles compared to a suspension in which particles consist of four graphene layers.

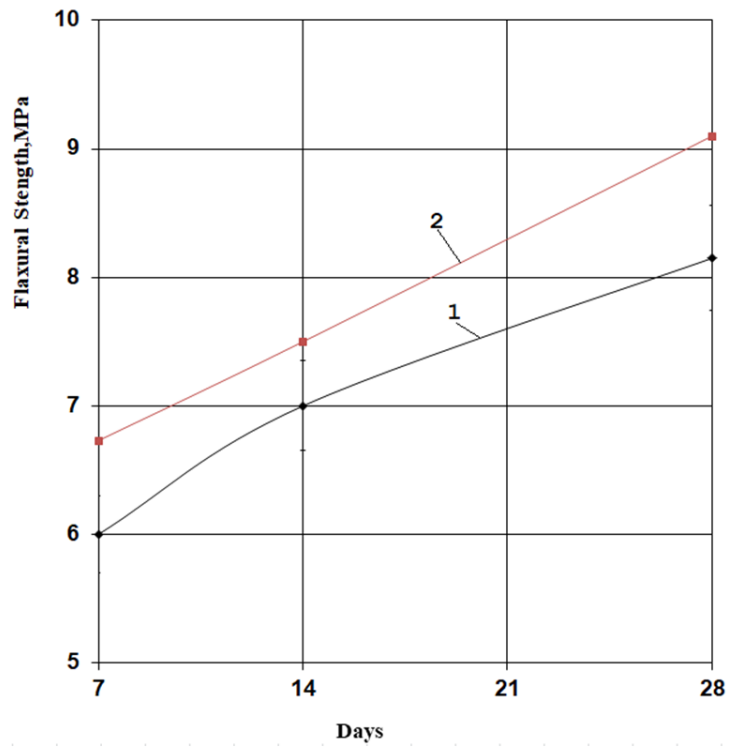

Fig. 3. Change in bending strength during curing: 1 - control images; 2 - modified with few-layer graphene. 


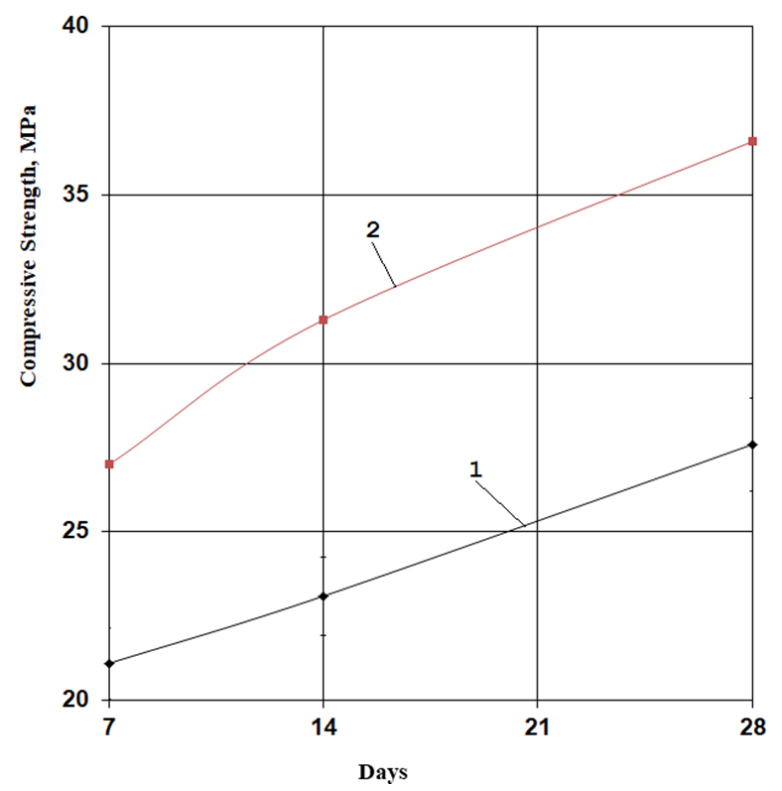

Fig. 4. Change in compressive strength in the curing process: 1 - control samples; 2 - modified with few-layer graphene.

\section{Conclusion}

It was experimentally established that the bending strength at three points of samples modified with graphene (graphene concentration of about 0.015 wt.\% With respect to cement) is about $16 \%$ higher than that of the original concrete, while the compressive strength is $33 \%$ higher. There is reason to assume that the main role in increasing the strength of concrete is played not by the mass concentration of few-layer graphene, but by the amount of its particles per unit volume. This assumption requires further detailed studies, because a decrease in the mass concentration of few-layer graphene in concrete, while maintaining its strength action, opens up a new path to the large-scale industrial use of environmentally friendly and inexpensive technology to improve the performance of concrete.

\section{References}

1. D. Dimov, I. Amit, O. Gorrie, M.D. Barnes, N.J. Townsend, A.I. Neves, F. Withers, S. Russo, M.F. Craciun, Adv. Funct. Mater. 28, 1705183 (2018)

2. D. Nivethitha, S.R. Srividhya, S. Dharmar, Int. J. Sci. Research, 5 (1), 913-916 (2016)

3. M.S. Morsy, S.H. Alsayed, M.A. Aqel, Int. J. Civ. \& Environ. Eng. 10 (01), 23-27 (2010)

4. G. Xu, S. Du, J. He, X. Shi, Carbon 148, 141-150 (2019)

5. D. Hou, Z. Lu, X. Li, H. Ma, Z. Li, Carbon 115, 188-208 (2017)

6. T.S. Qureshi, D.K. Panesar, B. Sidhureddy, A. Chen, P.C. Wood, Composites B 159, 248-258 (2019)

7. T.S. Qureshi, D.K. Panesar, Constr. Build. Mater. 206, 71-83 (2019) 
8. L. Zhao, X. Guo, Y. Liu, Y. Zhao, Z. Chen, Y. Zhang, L. Guo, X. Shu, J. Liu, Constr. Build. Mater. 190, 150-163 (2018)

9. M. Muthua, M. Santhanam, Cement Concrete Comp. 91, 118-137 (2018)

10. Q. Liu, Q. Xu, Q. Yu, R. Gao, T. Tong, Constr. Build. Mater. 127, 565-576 (2016)

11. A.P. Kauling, A.T. Seefeldt, D.P. Pisoni, R.C. Pradeep, R. Bentini, R.V.B. Oliveira, K.S. Novoselov, A.H.C. Neto, Adv. Mater. 30, 03784 (2018)

12. K.R. Paton, E. Varrla, C. Backes, R.J. Smith, U. Khan, A. O’Neill, C. Boland, M. Lotya, O.M. Istrate, P. King, T. Higgins, S. Barwich, P. May, P. Puczkarski, I. Ahmed, M. Moebius, H. Pettersson, E. Long, J. Coelho, S.E. O’Brien, E.K. McGuire, B.M. Sanchez, G.S. Duesberg, N. McEvoy, T.J. Pennycook, C. Downing, A. Crossley, V. Nicolosi, J.N. Coleman, Nat. mater. 13(6), 3944 (2014)

13. V. Pershin, K.A. Al-Shiblawi, A.M.R. Al-Mashhadani, A. Pasko, D. Melekhin, IOP Conf. Ser-Mat. Sci. 693(1), 012046 (2019) 